\title{
Progression from Acute Psychosis to Seizures and Autonomic In- stability in an Adolescent with Ovarian Mass
}

\author{
Andy Y Wen ${ }^{1 *}$, Jason Ziplow ${ }^{1}$, Benjamin Wadowski ${ }^{1}$ and Tanya Chadha ${ }^{2}$ \\ ${ }^{1}$ NYU School of Medicine, NYU Langone Medical Center, Bellevue Hospital Center, USA \\ ${ }^{2}$ Wolfson Children's Hospital, University of Florida College of Medicine, USA
}

*Corresponding author: Andy Y Wen, Department of Pediatrics, Division of Critical Care Medicine, NYU School of Medicine, NYU Langone Medical Center, Bellevue Hospital Center, 462 First Ave, NBV Suite 8E11, New York, NY 10016, USA, Tel: 212263-2377,Email: andy.wen@nyumc.org

\begin{abstract}
Anti-N-Methyl-D-Aspartate Receptor Encephalitis (NMDARE) is a complex clinical entity that is both diagnostically and therapeutically challenging. A 17-year-old girl presented to the emergency room with worsening anxiety, confusion, and acute psychosis. While in an inpatient psychiatric unit, she developed progressive catatonia with decreased responsiveness. After transfer to the pediatric inpatient unit, she became hyperreflexic with seizure-like activity consisting of back arching, left arm extension, and right arm flexion refractory to lorazepam. Electroencephalogram (EEG) monitoring was started on the first hospital day and within twenty-four hours showed extreme delta brush pattern and abnormal background activity. Cerebrospinal fluid analysis demonstrated pleocytosis with a lymphocytic predominance. Imaging studies were notable for a left ovarian mass and non-specific sulcal hyperintensity on magnetic resonance imaging of the brain. The ovarian mass was subsequently resected, and tumor pathology was consistent with a grade II immature teratoma. Serum and CSF NMDAR IgG antibody titers wereboth elevated to 1:320, which combined with extreme delta brush pattern on EEG confirmed the diagnosis of paraneoplastic NMDARE. Further treatment for the patient's NMDARE after tumor removal included intravenous immune globulin, high dose pulse steroids, plasmapheresis, and rituximab. The patient's hospital course was complicated by episodes of autonomic instability. Prevention of dysautonomic crises, agitation, and rigidity were managed with benzodiazepines, benztropine, and diphenhydramine. Antipyretics were given for unexplained fevers while multiple antihypertensive agents were given for episodic hypertension. Over several weeks, the patient demonstrated gradual neurocognitive improvement to near-baseline levels and was discharged to a rehabilitation facility for further recovery. NMDARE is a major cause of disease with both psychiatric and neurologic features.
\end{abstract}

Symptomatic treatment can be challenging requiring a balance of pharmacologic therapies. In the pediatric population, NMDARE is an important diagnosis to consider in applicable cases since it is a highly treatable disease with an overall good prognosis after adequate treatment.

\section{Keywords}

Encephalitis, Psychosis, Ovarian mass, Autonomic instability, Seizures
Abbreviations
CSF: Cerebro Spinal Fluid; EEG: Electroencephalogram; MRI: Magnetic Resonance Imaging; NMDA: N-Methyl-D-Aspartate; NMDARE: N-Methyl-D-Aspartate Receptor Encephalitis

\section{Introduction}

Anti-N-Methyl-D-Aspartate Receptor Encephalitis (NMDARE) is a type of autoimmune encephalitis that manifests with seizures, neuropsychiatric symptoms, and autonomic instability or central hypoventilation $[1,2]$. Recent case studies have found that risk factors for developing NMDARE include female gender, adolescence/young adulthood, as well as a possibility for viral infection/reactivation (e.g. herpes simplex virus and influenza A) [1]. The prevalence of this disorder has not been well identified, and large case series of children with NMDARE are rare in literature, however it has been reported to be the most common treatable form of autoantibody-mediated encephalitis [1-3].

Patients with concomitant tumor presence in NMDARE

Citation: Wen AY, Ziplow J, Wadowski B, Chadha T (2017) Progression from Acute Psychosis to Seizures and Autonomic Instability in an Adolescent with Ovarian Mass. Clin Med Rev Case Rep 4:174. doi. org/10.23937/2378-3656/1410174

Received: December 05, 2016: Accepted: June 28, 2017: Published: June 30, 2017

Copyright: (c) 2017 Wen AY, et al. This is an open-access article distributed under the terms of the Creative Commons Attribution License, which permits unrestricted use, distribution, and reproduction in any medium, provided the original author and source are credited. 
ranges from 6 to $50 \%$ and include immature testicular teratomas, lymphomas, or ovarian teratomas, with ovarian teratomas the most common [1]. In fact, of the $33 \%$ of adolescent girls who develops NMDARE with an associated teratoma, $30-50 \%$ of them will present with an ovarian teratoma that can be either unilateral or bilateral [1].

The pathophysiology of NMDARE is still under investigation, however it is known that the auto antibodies produced in this disease specifically target the NR1 and NR2 subunits of the N-Methyl-D-Aspartate (NMDA) receptors within the glutamatergic system, particularly the hypothalamus, limbic system, forebrain, and pituitary [1,2]. As a result of these auto antibodies, there is a loss of function of these receptors causing schizophrenia-like symptoms that includes cognitive and behavioral deficits (especially deficits in executive functioning and abstract thinking) as well as psychosis $[1,2]$.
Interestingly, both central nervous system tissues and ovarian tissues share similar proteins, perhaps explaining the production of auto antibodies to the NMDA receptors [1]. In addition, central nervous system tissue is itself found within teratomas, making it likely that the teratoma is not directly responsible for autoantibody production [1].

Out of all of the cases of NMDARE, approximately $40 \%$ of these are in patients less than 18 years of age with the youngest patient identified noted to be 8 -months-old [2]. Among pediatric cases under the age of $18,80 \%$ are young women [2], and among children under the age of $12,39 \%$ are male [4]. Importantly, pediatric patients that develop NMDARE, especially those of whom are prepubertal, usually present with neurologic symptoms over psychiatric symptoms, which is useful when considering NMDARE in a differential diagnosis [2].

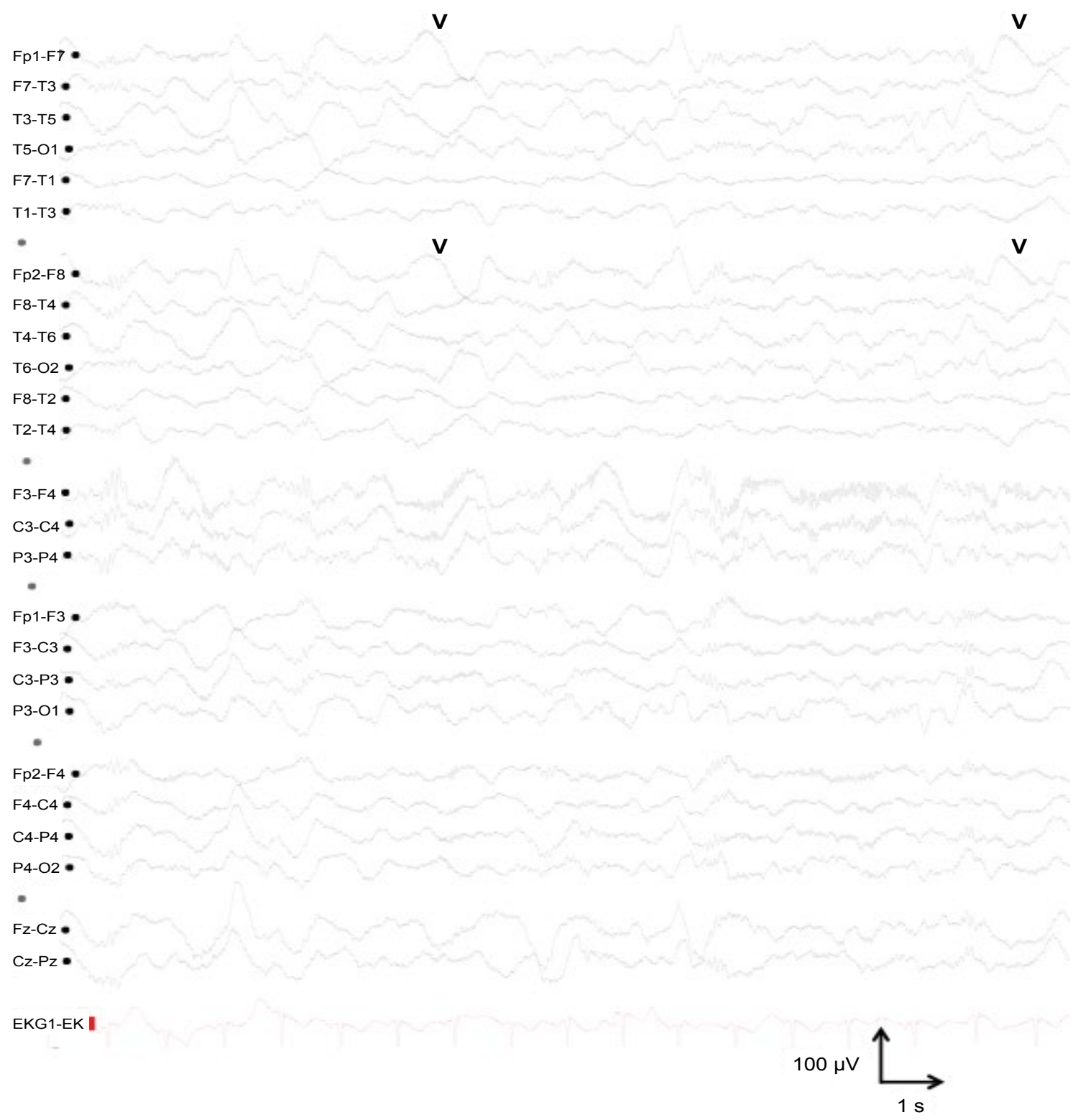

Figure 1: Continuous EEG recording on hospital day three demonstrating high-voltage beta activity superimposed on delta waves predominantly in the anterior areas consistent with extreme delta brush pattern (arrow heads). 


\section{Case Presentation}

A 17-year-old African American female presented with anxiety, confusion, and behavioral changes, and was admitted to an inpatient psychiatric unit. One month prior to presentation, she had developed anxiety after witnessing a stranger commit suicide. Subsequently, she began displaying signs of acute psychosis consisting of disorganized behavior, suicidal and homicidal ideation, fragmented speech, and visual hallucinations. During the first three hospital days, the patient had worsening anxiety and delirium that was treated intermittently with a combination of risperidone, lorazepam, thorazine, olanzapine, diphenhydramine, and haloperidol. By hospital day three, the patient whom had developed catatonia with poor oral intake, drooling, urinary retention, and unresponsiveness, was transferred to the inpatient pediatric floor for dehydration. On hospital day six the patient was unresponsive, hyperreflexic, and had bilateral ankle clonus on physical exam. On hospital day eight she began having multiple daily episodes of left arm extension, right arm flexion, and arching of the back each lasting several minutes each. These episodes were variable in that some were self-resolving while others required one or more doses of lorazepam prior to resolution. The remainder of her physical exam was unremarkable.

EEG monitoring, which was started on hospital day three when the patient was first admitted to the pediatric floor, initially showed diffuse abnormal background activity but on hospital day nine an extreme delta brush pattern (Figure 1) was observed that did not coincide with the patient's abnormal movements. The continuous EEG tracings were recorded in the context of having received lorazepam, thorazine, and riperidone the day before. Cerebrospinal Fluid (CSF) analysis on hospital day seven showed a white blood cell count of 21 cells/ $\mathrm{mm}^{3}$ (91\% lymphocytes), red blood cell count of 0 cells/ $\mathrm{mm}^{3}$, glucose $69 \mathrm{mg} / \mathrm{dL}$, protein $10.8 \mathrm{mg} / \mathrm{dL}$, IgG index 0.32 (normal 0.28-0.66), and seven oligoclonal bands not present in serum. The patient was started on acyclovir on hospital day seven for initial concern of viral meningoencephalitis. Magnetic Resonance Imaging (MRI) of the brain and spine was performed on hospital day nine and showed subtle sulcal FLAIR hyperintensity without evidence of meningeal enhancement, parenchymal signal abnormality, infarct, or hemorrhage. Additional serumand CSF studies for viral encephalitis, serum tumor markers, and paraneoplastic antibodies were negative, but IgG antibodies for NMDA receptors were identified in the serum (1:320) and in the CSF (1:320).

During the first month of the patient's hospital course, she remained unresponsive to verbal commands and had daily episodes of limb rigidity, autonomic instability with hypertensive crises, diaphoresis, ankle clonus, arching of the back, vocalizations, and facial stereotypies consisting of lip smacking, blinking, and grimacing. These episodes lasted for less than thirty minutes each and occurred with variable frequency between one and ten times daily. She was afebrile during these episodes. Out of concern for Neuroleptic Malignant Syndrome (NMS) antipsychotic medications were discontinued, and diphenhydramine and lorazepam were administered for symptomatic treatment. Throughout the remainder of her hospital course, the patient experienced occasional seizures treated acutely with lorazepam and fosphenytoin, and then long-term control was achieved with levetiracetam. She continued to have episodes of urinary retention and dystonia with a single episode of unresponsiveness and areflexia. Hypertension and episodes of hypertensive crisis were managed with amlodipine and clonidine, respectively. Additional workup included ultrasound and MRI of the pelvis showing a left-sided ovarian mass. Left salpingo-oophorectomy was performed and pathology report was consistent with grade II immature teratoma.

The differential diagnosis for this patient included NMS, Acute Demyelinating Encephalomyelitis (ADEM), and encephalitis secondary to a viral or autoimmune etiology. However, given the extreme delta brush pattern observed on EEG and with the presence of serum and CSF antibodies for NMDA receptors detected, the diagnosis of anti-NMDARE was confirmed. The patient was treated with Intravenous Immunoglobulin (IVIg) 1 $\mathrm{g} / \mathrm{kg} /$ day for two days on hospital day ten. Intravenous (IV) methylprednisolone $1 \mathrm{~g} /$ day was started on hospital day sixteen for three days. On hospital day nineteen, the patient clinically deteriorated and was not responsive to sternal rub. This prompted more aggressive treatment including left salpingo-oophorectomy on hospital day nineteen, plasmapheresis on hospital day twenty for five days, IVIg on hospital day twenty-seven, and then rituximab on hospital day thirty-five. Neurologic and cognitive function gradually improved following plasmapheresis and the second dose of IVIG. Immunotherapy was continued with rituximab on hospitals days forty-one, forty-eight, and sixty-two, and IV methylprednisolone 1 $\mathrm{g}$ on hospital days fifty-five and sixty-one. On hospital day thirty-nine the patient started to smile and make eye-contact, and at discharge to inpatient rehab on hospital day sixty-seven, the patient was functioning near her baseline levels. Over time the patient was able to communicate verbally, but did not remember much of her hospitalization. Neuropsychological evaluation performed over a year later showed that the patient had no speech or behavioral deficits, and cognition was grossly intact however deficits were noted on challenging tasks such as memory testing.

\section{Discussion}

NMDARE is a relatively new clinical entity initially described in 2005. In a case series of four women with ovarian teratoma, they were found to share certain clinical features including memory loss, alterations in consciousness, and psychiatric features. Later, it was 
discovered that each of these four patients had antibodies to the NMDA receptor in their CSF, a finding consistent with subsequent cases of this disease [5]. Thus, NMDARE has since become a clinically recognized type of paraneoplastic limbic encephalitis [6].

NMDARE has been identified as a triphasic disorder with a fairly characteristic time course. First, the patient will have a viral-like prodromal phase, which consists of such symptoms as headache, fever, and rhinitis [7]. Approximately two weeks from the prodromal phase, the second "early" phase begins resulting in cognitive dysfunction, psychiatric features, and seizures. The third "late" features usually begin 10-20 days after the early features and include reduced level of consciousness, involuntary movements, and autonomic dysfunction [8].

Although the prodromal stage of this disorder exists, initial presentations of these types of cases are often to psychiatric facilities due to the early psychiatric features and recognition of the prodromal phase only retrospectively. As many as 77 of 91 patients in a recent case series of NMDARE patients initially presented to psychiatric institutions with the psychiatric features of their illness [8]. Some of the psychiatric symptoms in these cases and others have included anxiety, agitation, bizarre behavior, delusional or paranoid thoughts, visual or auditory hallucinations, grandiosity, mania, mutism, and echolalia $[7,8]$. Many of these psychiatric manifestations were in fact exhibited by our patient during the initial course of her illness. Use of antipsychotic medications to control agitation, psychosis, and/or movement disorder in patients has been associated with neuroleptic malignant syndrome and therefore should be included in the differential [9].

In the later phase of the disease, patients commonly present with other neurologic changes. In a case series by De Nayer, et al. [8] 76\% of their patients developed seizures, $86 \%$ developed dyskinesias and other movement disorders, $66 \%$ had central hypoventilation and decreased level of consciousness occasionally requiring mechanical ventilation, and $69 \%$ had autonomic instability [8]. Symptoms of autonomic instability in these patients included such features as hyperthermia, tachycardia or bradycardia, hypertension or hypotension, and hypersalivation [7]. Of these, the seizures, dyskinesias (especially oro-facial), hypersalivation, and hypertension were prominent features of our patient's disease course.

It is important to remember that NMDARE is usually, although not always, a paraneoplastic phenomenon as this has an important role in affecting prognosis and treatment options in these patients. The detection of a tumor in association with NMDARE is dependent on age, sex, and ethnic background, and the younger the patient is the less likely a tumor will be detected [10]. The association of a tumor is known to be rare in pedi- atric patients, however in a recent study, $59 \%$ of cases were found to have associated neoplasms, and of these neoplasms 53 out of 58 of those were ovarian teratomas $[6,8]$. Epidemiological data collected from these cases have shown that ovarian teratomas in women with these disorders usually occur in older, black patients [7].

Definitive diagnosis of NMDARE is made primarily by detection of anti-NMDAR antibodies in the CSF or serum, and CSF anti-NMDAR antibodies are more sensitive than serum for NMDARE $[7,11]$. Of note, decreasing titers of the CSF NMDAR antibody often correlate with clinical improvement [5]. Although the association of ovarian teratomas with NMDARE has been increasingly recognized, the association is variable and their presence is not necessary to confirm the diagnosis [7].

NMDARE should be suspected in any patient presenting with acute onset of psychiatric symptoms, and therefore CSF anti-NMDAR antibody test should be sent to confirm the diagnosis. Additional studies including CSF serology and EEG, although not diagnostic, have characteristic features that are helpful in making the diagnosis of NMDARE. Anecdotal observations have shown that in the early phase of the disease CSF shows pleocytosis with lymphocytic predominance, whereas a finding of oligoclonal bands is more common in the later phase of this disease [5]. EEG activity in patients with NMDARE show generalized or predominantly frontotemporal delta-theta activity. During the psychotic stage (as opposed to the hyperkinetic stage), diffuse low voltage EEG activity seems to be predominant [12]. An important addition to these EEG findings in NMDARE is the extreme delta brush pattern, a pattern usually isolated to those demonstrated by premature infants, which may represent an important early clinical diagnostic entity in the future [13].

Treatment for NMDARE usually includes surgical removal if tumor is present, followed by first and occasionally second-line immunotherapy drugs [14]. The most common tumor removal is oophorocytectomy, and is usually followed by immunotherapy [6]. First line immunotherapy includes high dose IV corticosteroids, plasma exchange, and IV immunoglobulins [7]. In certain cases, especially in cases with delayed diagnoses, protracted courses, or those cases in patients without associated teratomas, second line therapies such as rituximab and cyclophosphamide have proven effective [7]. For instance a woman with NMDARE, but without an associated tumor was treated with rituximab successful after conventional immunotherapy consisting of corticosteroids, IVIg, and plasma exchange resulted in little improvement. In her case, significant improvement was noted approximately one week after rituximab administration demonstrating the potential efficacy of these second line treatments in refractory [15].

Prognosis for these patients varies, however studies have shown that approximately $75 \%$ of patients demon- 
strate complete or near-complete recoveries and that early treatment is a factor associated with good outcome $[4,7]$. Especially in cases with associated neoplasms and surgical resection of the malignancies with concomitant immunotherapy, relief of neurological and mental symptoms has been shown to occur on average $13.7 \pm 5.5$ days after surgery [6]. However, these patients may have persistent neurological disabilities resulting in subsequent mortality [16]. In these same studies, relapses consisting of symptom or neoplasm recurrence occurred months to years after initial resolution in $25 \%$ of patients [7]. Interestingly, patients without associated tumor have been shown to have increased chances of relapse on average compared to those with surgically removed tumors [5].

\section{Conclusion}

NMDARE represents a complex clinical entity that is becoming increasingly recognized as a major cause of disease with combined psychiatric and neurological features. In the pediatric population, it is important to keep NMDARE on the list of differential diagnoses in applicable cases since it is a highly treatable disease with an overall good prognosis after adequate treatment. Diagnosis of this disease currently includes primarily antibody testing, but future diagnoses could be made using characteristic EEG findings or other classic clinical criteria.

\section{Acknowledgements}

The authors thank Dr. Daniel Miles and Nidia Ortiz for technical assistance.

\section{Funding Source}

No funding was secured for this study.

\section{Financial Disclosures}

The authors have no financial relationships relevant to the article to disclose.

\section{Conflict of Interest}

The authors have no conflicts of interest to disclose.

\section{Contributor's Statement}

Andy Y. Wen: Dr. Wen drafted the initial manuscript, critically reviewed the manuscript, and approved the final manuscript as submitted. Jason Ziplow: Dr. Ziplow drafted the initial manuscript, and approved the final manuscript as submitted. Benjamin Wadowski: Mr. Wadowski drafted, reviewed, and revised the initial manuscript, and approved the final manuscript as submitted. Tanya Chadha: Dr. Chadha drafted, reviewed, and revised the initial manuscript, and approved the final manuscript as submitted.

\section{References}

1. Scheer S, John RM (2016) Anti-N-Methyl-D-Aspartate Receptor Encephalitis in Children and Adolescents. J Pediatr Health Care 30: 347-358.

2. Brenton JN, Kim J, Schwartz RH (2016) Approach to the Management of Pediatric-Onset Anti-N-Methyl-d-Aspartate
(Anti-NMDA) Receptor Encephalitis: A Case Series. J Child Neurol 31: 1150-1155.

3. Sartori S, Nosadini M, Cesaroni E, Falsaperla R, Capovilla G, et al. (2015) Paediatric anti-N-methyl-D-aspartate receptor encephalitis: The first Italian multicenter case series. Eur J Paediatr Neurol 19: 453-463.

4. Titulaer MJ, Mc Cracken L, Gabilondo I, Armangué T, Glaser C, et al. (2013) Treatment and prognostic factors for long-term outcome in patients with anti-NMDA receptor encephalitis: an observational cohort study. Lancet Neurol 12: 157-165.

5. Sharma B, Handa R, Prakash S, Nagpal K, Gupta P (2014) Anti-NMDA receptor encephalitis: a neurological disease in psychiatric disguise. Asian J Psychiatr 7: 92-94.

6. Bai Y, Guan Q, Jiang J, Zhang Z (2016) Treatment principles of ovarian teratoma with anti- $\mathrm{N}$-methyl-D-aspartate receptor encephalitis. Arch Gynecol Obstet 294: 623-629.

7. Howard CM, Kass JS, Bandi VDP, Guntupalli KK (2014) Challenges in providing critical care for patients with anti-N-methyl-D-aspartate receptor encephalitis. Chest 145 : 1143-1147.

8. De Nayer AR, Myant N, Sindic CJ (2009) A subacute behavioral disorder in a female adolescent. Autoimmune anti-N-methyl-D-aspartate receptor encephalitis associated with ovarian teratoma. Biol Psychiatry 66: 13-14.

9. Mohammad SS, Jones $H$, Hong M, Nosadini M, Sharpe C, et al. (2016) Symptomatic treatment of children with anti-NMDAR encephalitis. Dev Med Child Neurol 58: 376-384.

10. Dalmau J, Lancaster E, Martinez-Hernandez E, Rosenfeld MR, Balice-Gordon R (2011) Clinical experience and laboratory investigations in patients with anti-NMDAR encephalitis. The Lancet Neurology 10: 63-74.

11. Mirza MK, Pogoriler J, Paral K, Ananthanarayanan V, Mandal $S$, et al. (2011) Adjunct therapeutic plasma exchange for anti-N-methyl-D-aspartate receptor antibody encephalitis: a case report and review of literature. J Clin Apher 26: 362-365.

12. Kataoka H, Takatani T, Ueno S (2012) Low-voltage EEG activity presenting from psychotic stage in a patient with anti-NMDA receptor encephalitis. BMJ Case Rep.

13. Soylemez E, Güveli BT, Atakli D, Yatmazoğlu M, Atay T, et al. (2015) Extreme Delta Brush Eeg Pattern in a Case with Anti-Nmda Receptor Encephalitis. Ideggyogy Sz 68: 357-360.

14. Gastaldi M, Thouin A, Vincent A (2016) Antibody-Mediated Autoimmune Encephalopathies and Immunotherapies. Neurotherapeutics 13: 147-162.

15. Ikeguchi R, Shibuya K, Akiyama S, Hino S, Kubo H, et al. (2012) Rituximab used successfully in the treatment of anti-NMDA receptor encephalitis. Intern Med 51: 1585-1589.

16. Dalmau J, Gleichman AJ, Hughes EG, Rossi JE, Peng X, et al. (2008) Anti-NMDA-receptor encephalitis: case series and analysis of the effects of antibodies. Lancet Neurol 7: 1091-1098. 